\title{
IDEMPOTENCE-PRESERVING MAPS BETWEEN MATRIX SPACES OVER FIELDS OF CHARACTERISTIC 2*
}

\author{
JIN-LI XU ${ }^{\dagger}, \mathrm{XIAO}-\mathrm{MIN}^{\mathrm{TANG}^{\dagger}}{ }^{\text {, AND }}$ CHONG-GUANG $\mathrm{CAO}^{\dagger}$
}

\begin{abstract}
Let $M_{n}(\mathbb{F})$ be the space of all $n \times n$ matrices over a field $\mathbb{F}$ of characteristic 2 other than $\mathbb{F}_{2}=\{0,1\}$, and let $P_{n}(\mathbb{F})$ be the subset of $M_{n}(\mathbb{F})$ consisting of all $n \times n$ idempotent matrices. Let $m$ and $n$ be integers with $n \geq m$ and $n \geq 3$. We denote by $\Phi_{n, m}(\mathbb{F})$ the set of all maps from $M_{n}(\mathbb{F})$ to $M_{m}(\mathbb{F})$ satisfying that $A-\lambda B \in P_{n}(\mathbb{F})$ implies $\phi(A)-\lambda \phi(B) \in P_{m}(\mathbb{F})$ for all $A, B \in M_{n}(\mathbb{F})$ and $\lambda \in \mathbb{F}$. In this paper, we give a complete characterization of $\Phi_{n, m}(\mathbb{F})$.
\end{abstract}

Key words. Field; Characteristic; Idempotence; Preserving; Homogeneous

AMS subject classifications. 15A04.

1. Introduction. Suppose $\mathbb{F}$ is an arbitrary field. Let $M_{n}(\mathbb{F})$ be the space of all $n \times n$ matrices over $\mathbb{F}$ and $P_{n}(\mathbb{F})$ be the subset of $M_{n}(\mathbb{F})$ consisting of all $n \times n$ idempotent matrices. Denote by $E_{i j}$ the $n \times n$ matrix which has 1 in the $(i, j)$ entry and has 0 elsewhere. For any positive integer $k \leq n$, let $\mathbb{F}^{k}$ be the vector space of all $k \times 1$ matrices over $\mathbb{F}$. Let $e_{1}, e_{2}, \ldots, e_{n}$ denote the vectors of the canonical basis of $\mathbb{F}^{n}$. We denote by $I_{k}$ and $0_{k}$ the $k \times k$ identity matrix and zero matrix, respectively, or simply $I$ and 0 , if the dimensions of these matrices are clear.

The problem of characterizing linear maps preserving idempotence belongs to a large group of the so-called linear preserver problems (see [3] and the references therein). The theory of linear preservers of idempotence is well-developed (see [1, 4]). Some initial results on more difficult non-linear idempotence preserver problems have been obtained $[5,2,8]$. We denote by $S \Phi_{n}(\mathbb{F})$ the set of all maps from $M_{n}(\mathbb{F})$ to itself satisfying that $A-\lambda B \in P_{n}(\mathbb{F}) \Longleftrightarrow \phi(A)-\lambda \phi(B) \in P_{n}(\mathbb{F})$ for all $A, B \in M_{n}(\mathbb{F})$ and $\lambda \in \mathbb{F}$. A map $\phi$ is called a strong idempotence-preserving map if $\phi \in S \Phi_{n}(\mathbb{F})$. Šemrl [5], Dolinar [2] and Zhang [8] characterize the set of strong idempotence-preserving maps $S \Phi_{n}(\mathbb{F})$, where $\mathbb{F}$ is a field of characteristic other than 2 .

Recently, Tang et. al. [6] improve the results mentioned above by characterizating

${ }^{*}$ Received by the editors on June 10, 2009. Accepted for publication on July 31, 2010. Handling Editors: Roger A. Horn and Fuzhen Zhang.

$\dagger$ Department of Mathematics, Heilongjiang University, Harbin, 150080, P. R. China (x.m.tang@163.com). This work is Project 10671026 supported by National Natural Science Foundation of China, Postdoctoral Scientific Research Foundation of Heilongjiang Province (no. HB200801165) and the fund of Heilongjiang Education Committee (no. 11541268). J.L. Xu is supported by Youth Foundation of Heilongjiang University. 
of the set $\Phi_{n}(\mathbb{F})$ of idempotence-preserving maps from $M_{n}(\mathbb{F})$ to itself satisfying that $A-\lambda B \in P_{n}(\mathbb{F})$ implies $\phi(A)-\lambda \phi(B) \in P_{n}(\mathbb{F})$ for all $A, B \in M_{n}(\mathbb{F})$ and $\lambda \in \mathbb{F}$. However, they are still confined to the fields of characteristic other than 2. Tang et. al. [7] studies the same problem over fields $\mathbb{F}$ of characteristic 2 except $\mathbb{F}_{2}=\{0,1\}$, under the assumption that there exists an invertible matrix $T \in M_{n}(\mathbb{F})$ such that $T \phi\left(E_{k k}\right) T^{-1}=E_{k k}$ for all $k \in\{1, \ldots, n\}$. In this paper, we consider the remaining problem between spaces having different dimensions.

Let $m$ and $n$ be integers with $n \geq m$ and $n \geq 3$. We denote by $\Phi_{n, m}(\mathbb{F})$ the set of all maps from $M_{n}(\mathbb{F})$ to $M_{m}(\mathbb{F})$ for which $A-\lambda B \in P_{n}(\mathbb{F})$ implies $\phi(A)-\lambda \phi(B) \in$ $P_{m}(\mathbb{F})$ for all $A, B \in M_{n}(\mathbb{F})$ and $\lambda \in \mathbb{F}$. We will characterize the set $\Phi_{n, m}(\mathbb{F})$ when the field $\mathbb{F}$ is of characteristic 2 and $\mathbb{F} \neq \mathbb{F}_{2}$. Hence, the result of this paper complements the results of $[6]$.

Since the field we consider is of characteristic 2,2 does not have a multiplicative inverse. Hence, the approach of the above mentioned references does not work. In fact, if the field is of characteristic 2, then the problem is more complicated. To overcome the difficulties, the following two new ideas are pivotal:

(i) We define a string of subsets $\Delta_{n, k, \mu}$ of $M_{n}(\mathbb{F})$. Then we use the subsets $\Delta_{n, k, \mu}$ to prove some result by induction. The string of subsets $\Delta_{n, k, \mu}$ is interesting itself.

(ii) The images of $E_{i i}$ under $\phi$ are important for our purpose. But the cases of $\phi\left(E_{i i}\right)$ are complicated. We show that $\phi\left(E_{i i}\right)$ may take one of three distinct forms (see Lemma 3.3). This is different from the case of characteristic other than 2 .

2. Characterization of some subsets of $M_{n}(\mathbb{F})$. In the rest of this paper, we always let $m$ and $n$ be integers with $n \geq m$ and $n \geq 3$ unless otherwise stated, and let $\mathbb{F}$ be a field of characteristic 2 other than $\mathbb{F}_{2}$. For $x \in \mathbb{F}^{n} \backslash\{0\}$, we denote $S_{n, x}=\left\{P \in P_{n}(\mathbb{F}): P x \neq x\right\}$. Next, we define by induction on $k$ a string of sets $\Delta_{n, k, \mu}$ as follows for every $\mu \in \mathbb{F}^{*}$, where $\mathbb{F}^{*}=\mathbb{F} \backslash\{0\}$.

(i) $\Delta_{n, 0, \mu}=\left\{0 \in M_{n}(\mathbb{F})\right\}$;

(ii) $\Delta_{n, k, \mu}=\left\{A \in M_{n}(\mathbb{F})\right.$ : there are $B \in \Delta_{n, k-1, \mu}$ and $\lambda \in \mathbb{F}^{*} \backslash\left\{\mu^{-1}\right\}$ such that $\left.\lambda A+B \in P_{n}(\mathbb{F})\right\}$ for $1 \leq k \leq 2 n^{2}$.

The following lemma is useful for the proof of our main theorem.

Lemma 2.1. ([7]) For any fixed $\mu \in \mathbb{F}^{*}$, we have $M_{n}(\mathbb{F})=\cup_{k=0}^{2 n^{2}} \Delta_{n, k, \mu}$.

3. Preliminary results. This section provides some preliminary results. 
Lemma 3.1. ([7]) If $\phi \in \Phi_{n, m}(\mathbb{F})$, then

(i) $\phi\left(P_{n}(\mathbb{F})\right) \subseteq P_{m}(\mathbb{F})$;

(ii) $\phi$ is homogeneous, i.e., $\phi(\lambda A)=\lambda \phi(A)$ for every $A \in M_{n}(\mathbb{F})$ and $\lambda \in \mathbb{F}$.

Lemma 3.2. Suppose that $\phi \in \Phi_{n, m}(\mathbb{F})$ and $A, B \in P_{n}(\mathbb{F})$ satisfy $A+B \in P_{n}(\mathbb{F})$. Then

$$
\phi(A+\lambda B)=\phi(A)+\lambda \phi(B) \text { for every } \lambda \in \mathbb{F} .
$$

Proof. For any $\lambda \in \mathbb{F} \backslash\{0,1\}$, since $(A+\lambda B)+\lambda B,(A+\lambda B)+(1+\lambda) B, \lambda^{-1}(A+$ $\lambda B)+\lambda^{-1} A, \lambda^{-1}(A+\lambda B)+\left(1+\lambda^{-1}\right) A$ are idempotent, by $\phi \in \Phi_{n, m}(\mathbb{F})$ and (ii) of Lemma 3.1, we deduce:

$$
\begin{gathered}
\phi(A+\lambda B)+\lambda \phi(B) \in P_{m}(\mathbb{F}), \\
\phi(A+\lambda B)+(1+\lambda) \phi(B) \in P_{m}(\mathbb{F}), \\
\lambda^{-1}[\phi(A+\lambda B)+\phi(A)] \in P_{m}(\mathbb{F}), \\
\lambda^{-1} \phi(A+\lambda B)+\left(1+\lambda^{-1}\right) \phi(A) \in P_{m}(\mathbb{F}) .
\end{gathered}
$$

Applying Lemma 3.1 (i) to $B \in P_{n}(\mathbb{F})$, we have $\phi(B) \in P_{m}(\mathbb{F})$, so we deduce from (3.1) and (3.2) that

$$
\phi(A+\lambda B) \phi(B)+\phi(B) \phi(A+\lambda B)=0 .
$$

This, together with (3.1), gives that

$$
\phi(A+\lambda B)^{2}=\phi(A+\lambda B)+\lambda(\lambda+1) \phi(B) .
$$

Similarly, one has by (3.3), (3.4) and $\phi(A)^{2}=\phi(A)$ that

$$
\phi(A+\lambda B)^{2}=\lambda \phi(A+\lambda B)+(\lambda+1) \phi(A) .
$$

Using (3.5) and (3.6) and noticing that $\lambda \neq 1$, we have

$$
\phi(A+\lambda B)=\phi(A)+\lambda \phi(B) \text { for every } \lambda \in \mathbb{F} \backslash\{0,1\} .
$$

Since $\lambda \neq 0,1$, we see that $\lambda+1 \neq 0,1$. Also, we have $A+B, B,(A+B)+B$ are idempotent. This, together with (3.7), implies that

$$
\phi(A+\lambda B)=\phi((A+B)+(\lambda+1) B)=\phi(A+B)+(\lambda+1) \phi(B) .
$$


It follows from (3.7) and (3.8) that $\phi(A+B)=\phi(A)+\phi(B)$. We get the desired conclusion.

Lemma 3.3. Suppose $\phi \in \Phi_{n, m}(\mathbb{F})$. Then there exists an invertible matrix $T \in M_{m}(\mathbb{F})$ such that one of the following holds:

(a) $m=n$ and $T \phi\left(E_{k k}\right) T^{-1}=E_{k k}$ for all $k \in\{1, \ldots, n\}$;

(b) $m=n$ and $T \phi\left(E_{k k}\right) T^{-1}=E_{k k}+I_{n}$ for all $k \in\{1, \ldots, n\}$;

(c) There is an $r \in\{0,1, \ldots, m\}$ such that $T \phi\left(E_{k k}\right) T^{-1}=I_{r} \oplus 0_{m-r}$ for all $k \in\{1, \ldots, n\}$ (here $I_{r} \oplus 0_{m-r}=I_{m}$ if $r=m$, or 0 if $r=0$ ).

Proof. The proof is divided into three steps.

Step 1. There are an invertible matrix $Q_{0} \in M_{m}(\mathbb{F})$ and $\varepsilon_{i j} \in\{0,1\}, i=$ $1,2, \ldots, m, j=1,2, \ldots, n$, such that

$$
\phi\left(E_{k k}\right)=Q_{0} \operatorname{diag}\left(\varepsilon_{1 k}, \varepsilon_{2 k}, \ldots, \varepsilon_{m k}\right) Q_{0}^{-1} \text { for all } k \in\{1, \ldots, n\} .
$$

In fact, for any distinct $1 \leq i, j \leq n$, because of $E_{i i}, E_{j j}, E_{i i}+E_{j j} \in P_{n}(\mathbb{F})$, it follows from $\phi \in \Phi_{n, m}(\mathbb{F})$ that $\phi\left(E_{i i}\right), \phi\left(E_{j j}\right), \phi\left(E_{i i}\right)+\phi\left(E_{j j}\right) \in P_{n}(\mathbb{F})$. Hence $\phi\left(E_{i i}\right) \phi\left(E_{j j}\right)=\phi\left(E_{j j}\right) \phi\left(E_{i i}\right)$. It is easy to see that the claim in Step 1 holds.

For convenience, we assume by Step 1 that $\phi\left(E_{k k}\right)=\operatorname{diag}\left(\varepsilon_{1 k}, \varepsilon_{2 k}, \ldots, \varepsilon_{m k}\right)$ for all $k \in\{1, \ldots, n\}$. Let $E$ denote the $m \times n$ matrix $\left[\varepsilon_{i j}\right]$ with entries for $\{0,1\}$. Let

$$
L_{k}(E)=\left\{i: \varepsilon_{i k}=1\right\}, R_{k}(E)=\left\{i: \varepsilon_{i k}=0\right\}, k=1,2, \ldots, n .
$$

For $\pi \subset\{1, \ldots, n\}$ with $|\pi| \geq 2$, we define

$L_{k}^{\pi}(E)=L_{k}(E) \cap\left(\cap_{i \in \pi \backslash\{k\}} R_{i}(E)\right), R_{k}^{\pi}(E)=R_{k}(E) \cap\left(\cap_{i \in \pi \backslash\{k\}} L_{i}(E)\right)$ for all $k \in \pi$.

Step 2. Suppose that $\pi \subset\{1, \ldots, n\}$ with $|\pi| \geq 2$. Then

(i) $\left|L_{i}^{\pi}(E)\right|=\left|L_{j}^{\pi}(E)\right|$ for any $i, j \in \pi$;

(ii) $\left|R_{i}^{\pi}(E)\right|=\left|R_{j}^{\pi}(E)\right|$ for any $i, j \in \pi$.

Take distinct $i, j \in \pi$. For convenience, we let $r_{1}=\left|L_{i}^{\pi}(E)\right|, r_{2}=\left|L_{j}^{\pi}(E)\right|$, $s=\left|\cap_{k \in \pi} L_{k}(E)\right|, t=\left|\cap_{k \in \pi} R_{k}(E)\right|$ and $u=m-r_{1}-r_{2}-s-t$. Then there are a permutation matrix $Q$ and $\zeta_{1 p}, \ldots, \zeta_{\text {up }} \in\{0,1\}, p \in \pi$ such that

$$
\begin{gathered}
Q^{-1} \phi\left(E_{i i}\right) Q=I_{r_{1}} \oplus 0_{r_{2}} \oplus I_{s} \oplus 0_{t} \oplus \operatorname{diag}\left(\zeta_{1 i}, \ldots, \zeta_{u i}\right), \\
Q^{-1} \phi\left(E_{j j}\right) Q=0_{r_{1}} \oplus I_{r_{2}} \oplus I_{s} \oplus 0_{t} \oplus \operatorname{diag}\left(\zeta_{1 j}, \ldots, \zeta_{u j}\right),
\end{gathered}
$$


and

$Q^{-1} \phi\left(E_{k k}\right) Q=0_{r_{1}} \oplus 0_{r_{2}} \oplus I_{s} \oplus 0_{t} \oplus \operatorname{diag}\left(\zeta_{1 k}, \ldots, \zeta_{u k}\right)$, for all $k \in \pi \backslash\{i, j\}$ if $|\pi|>2$

Take $\lambda \neq 0,1$. Note that $(1+\lambda)^{-1}\left(E_{i i}+\lambda E_{i j}\right)+(1+\lambda)^{-1} \lambda E_{i i}, E_{i i}$ and $E_{i i}+\lambda E_{i j}$ are idempotent. This, together with Lemma 3.1 and $\phi \in \Phi_{n, m}(\mathbb{F})$, imply that

$$
\phi\left(E_{i i}+\lambda E_{i j}\right)=\phi\left(E_{i i}\right)+\phi\left(E_{i i}\right) \phi\left(E_{i i}+\lambda E_{i j}\right)+\phi\left(E_{i i}+\lambda E_{i j}\right) \phi\left(E_{i i}\right)
$$

Let $X$ denote the matrix $\phi\left(E_{i i}\right) \phi\left(E_{i i}+\lambda E_{i j}\right)+\phi\left(E_{i i}+\lambda E_{i j}\right) \phi\left(E_{i i}\right)$. By $\phi\left(E_{i i}\right), \phi\left(E_{i i}+\right.$ $\left.\lambda E_{i j}\right) \in P_{m}(\mathbb{F})$ and $(3.11)$, we deduce that

$$
X^{2}=0
$$

and

$$
X=\phi\left(E_{i i}\right) X+X \phi\left(E_{i i}\right) .
$$

Applying Lemma 3.2 to $E_{i i}, E_{j j}, E_{i i}+E_{j j} \in P_{n}(\mathbb{F})$, we have

$$
\phi\left(E_{i i}+E_{j j}\right)=\phi\left(E_{i i}\right)+\phi\left(E_{j j}\right) .
$$

Because of $\left(E_{i i}+\lambda E_{i j}\right)+\left(E_{i i}+E_{j j}\right) \in P_{n}(\mathbb{F})$, we have by (3.11), (3.14), Lemma 3.2 and $\phi \in \Phi_{n, m}(\mathbb{F})$ that

$$
\phi\left(E_{j j}\right)+\phi\left(E_{i i}\right) \phi\left(E_{i i}+\lambda E_{i j}\right)+\phi\left(E_{i i}+\lambda E_{i j}\right) \phi\left(E_{i i}\right) \in P_{m}(\mathbb{F}) .
$$

This, together with $(3.12)$ and the fact $\phi\left(E_{j j}\right) \in P_{m}(\mathbb{F})$, yields that

$$
X=\phi\left(E_{j j}\right) X+X \phi\left(E_{j j}\right)
$$

We now can assume by (3.9), (3.10), (3.13) and (3.15) that

$$
Q^{-1} X Q=\left[\begin{array}{ccccc}
0_{r_{1}} & X_{12} & 0 & 0 & X_{15} \\
X_{21} & 0_{r_{2}} & 0 & 0 & X_{25} \\
0 & 0 & 0_{s} & X_{34} & X_{35} \\
0 & 0 & X_{43} & 0_{t} & X_{45} \\
X_{51} & X_{52} & X_{53} & X_{54} & X_{55}
\end{array}\right] \text {, where } X_{55} \in M_{u}(\mathbb{F})
$$

If $|\pi|=2$, then $u=0$, so that

$$
Q^{-1} X Q=\left[\begin{array}{cc}
0_{r_{1}} & X_{12} \\
X_{21} & 0_{r_{2}}
\end{array}\right] \oplus\left[\begin{array}{cc}
0_{s} & X_{34} \\
X_{43} & 0_{t}
\end{array}\right]
$$


In the other case, $|\pi| \geq 3$. We claim that $X_{15}=0, X_{25}=0, X_{51}=0$ and $X_{52}=0$.

In fact, if $X_{15} \neq 0$, then there is a $(p, q)$ entry $x_{p q} \neq 0$ of $X_{15}$. And hence we see by (3.13) and (3.15) that $\zeta_{q i}=0$ and $\zeta_{q j}=1$. By the definition of $L_{j}^{\pi}(E)$, one can conclude that there is a $k \in \pi \backslash\{i, j\}$ such that $\zeta_{q k}=1$. Note that $E_{k k}+\left(E_{i i}+\lambda E_{i j}\right) \in$ $P_{n}(\mathbb{F})$. This, together with Lemma 3.2, (3.11), (3.12) and $\phi \in \Phi_{n, m}(\mathbb{F})$, yields that

$$
X=\left(\phi\left(E_{k k}\right)+\phi\left(E_{i i}\right)\right) X+X\left(\phi\left(E_{k k}\right)+\phi\left(E_{i i}\right)\right) .
$$

By a direct computation, we get $x_{p q}=0$, which is impossible. Similarly, we have $X_{25}=0, X_{51}=0$ and $X_{52}=0$. Thus,

$$
Q^{-1} X Q=\left[\begin{array}{cc}
0_{r_{1}} & X_{12} \\
X_{21} & 0_{r_{2}}
\end{array}\right] \oplus\left[\begin{array}{ccc}
0_{s} & X_{34} & X_{35} \\
X_{43} & 0_{t} & X_{45} \\
X_{53} & X_{54} & X_{55}
\end{array}\right] .
$$

By composing (3.9), (3.11) with (3.16) or (3.17), one can assume that

$$
Q^{-1} \phi\left(E_{i i}+\lambda E_{i j}\right) Q=\left[\begin{array}{cc}
I_{r_{1}} & X_{12} \\
X_{21} & 0_{r_{2}}
\end{array}\right] \oplus Y, \text { where } Y \in M_{m-r_{1}-r_{2}}(\mathbb{F}) .
$$

Take $\sigma \neq 0,1$ and $\lambda=\sigma^{-1}(\sigma+1)$. This, together with (3.18), allows us to assume that

$$
Q^{-1} \phi\left(\sigma E_{i i}+(1+\sigma) E_{i j}\right) Q=\left[\begin{array}{cc}
\sigma I_{r_{1}} & A \\
B & 0_{r_{2}}
\end{array}\right] \oplus U
$$

where $U \in M_{m-r_{1}-r_{2}}(\mathbb{F})$.

By a similar argument, we can assume that

$$
Q^{-1} \phi\left(\sigma E_{j i}+(1+\sigma) E_{j j}\right) Q=\left[\begin{array}{cc}
0_{r_{1}} & C \\
D & (\sigma+1) I_{r_{2}}
\end{array}\right] \oplus V
$$

where $V \in M_{m-r_{1}-r_{2}}(\mathbb{F})$.

Note that $\left(\sigma E_{i i}+(1+\sigma) E_{i j}\right)+\left(\sigma E_{j i}+(1+\sigma) E_{j j}\right) \in P_{n}(\mathbb{F})$. This, together with (3.19), (3.20) and $\phi \in \Phi_{n, m}(\mathbb{F})$, yields that

$$
\left[\begin{array}{cc}
\sigma I_{r_{1}} & A+C \\
B+D & (\sigma+1) I_{r_{2}}
\end{array}\right] \in P_{r_{1}+r_{2}}(\mathbb{F}) .
$$

If $r_{1}=0$ but $r_{2} \neq 0$, then we have by (3.21) that $(\sigma+1) I_{r_{2}} \in P_{r_{2}}(\mathbb{F})$, which is a contradiction. So $r_{1}=0$ implies $r_{2}=0$. Similarly, $r_{2}=0$ also implies $r_{1}=0$. We now consider the case $r_{1} \neq 0$ and $r_{2} \neq 0$. By (3.21) one has

$$
\sigma(\sigma+1) I_{r_{1}}=(A+C)(B+D), \sigma(\sigma+1) I_{r_{2}}=(B+D)(A+C) .
$$


This tells us that $r_{1}=r_{2}$. Thus, $\left|L_{i}^{\pi}(E)\right|=\left|L_{j}^{\pi}(E)\right|$. Similarly, we have $\left|R_{i}^{\pi}(E)\right|=$ $\left|R_{j}^{\pi}(E)\right|$. By the arbitrariness of $i, j$, we complete the proof of Step 2.

Let $\pi=\left\{l_{1}, \ldots, l_{t}\right\} \subset\{1, \ldots, n\}$, and let $F$ be the submatrix of $E$ consisting only of columns $l_{1}, \ldots, l_{t}$ of $E$. Before Step 3, we state the following two remarks.

Remark I. Due to $(i)$ of Step 2, we see that if there is an entry equal to 1 and all others equal to 0 in a proper row of $F$, then $F$ consists a $t \times t$ permutation matrix as its submatrix.

Remark II. Due to $(i i)$ of Step 2, we see that if there is an entry equal to 0 and all others equal to 1 in a proper row of $F$, then $F$ consists a submatrix $W+J$, where $W$ is a $t \times t$ permutation matrix and $J$ is a $t \times t$ matrix in which all entries are 1 . E.

Step 3. We will prove the conclusion based on the distribution of $0 \mathrm{~s}$ and $1 \mathrm{~s}$ in

If all columns of $E$ are the same, then we can easily check that $(c)$ holds. Otherwise, there is not only 0 but 1 in a certain row of $E$. Let $r$ be the largest number of zeros in a nonzero row; let $e$ denote such a row. If $r=n-1$, then let $\pi=\{1, \ldots, n\}$, and so we see by Remark I that ( $a$ ) holds. In the other case, we have $n \geq 3$. We can obtain a matrix $E_{1}$ from $E$ by first making a row permutation so that $e$ is its first row, and then by making a finite number of column permutations, so that $E_{1}$ is of the form

$$
E_{1}=\left[\begin{array}{ccccccc}
1 & 0 & \ldots & 0 & 1 & \ldots & 1 \\
* & * & \ldots & * & * & \ldots & *
\end{array}\right]
$$

where $*$ denotes any matrix of the appropriate size. For $E_{1}$ we take $\pi=\{1, \ldots, r+1\}$. Using Remark I, one can obtain a matrix $E_{2}=\left[\begin{array}{cc}I_{r+1} & B \\ C & D\end{array}\right]$ from $E_{1}$ by a finite number proper row permutations. Since $r$ is the biggest, we see that all entries of $B$ are 1. Furthermore, consider the submatrix $S$ of $E_{2}$ consisting of $r+1$-th, $\ldots, n$-th columns of $E_{2}$. Then it is clear that the first row of $S$ has an entry equal to 0 and all others equal to 1 . For $E_{2}$, we take $\pi=\{r+1, r+2, \ldots, n\}$. Using Remark II, one can obtain a matrix $E_{3}=\left[\begin{array}{cc}I_{r+1} & B \\ C_{1} & I_{n-r-1}+J\end{array}\right]$ from $E_{2}$ by a finite number proper row permutations, where $J \in M_{n-r-1}(\mathbb{F})$ has all entries equal to 1 and $C_{1}$ has all entries of its last column equal to 1 . By $m \leq n$, one has $m=n$. For $E_{3}$ we take $\pi=\{r+1, r+2\}$. It follows from $\left|L_{r+1}^{\pi}(E)\right|=\left|L_{r+2}^{\pi}(E)\right|$ that $r=1$. This means that in the first row of $E$ there is an entry equal to 0 and all other entries are equal to 1 . Finally, it follows by Remark II that $E$ is a sum of a permutation matrix and a matrix in which all entries are 1 , which implies $(b)$.

Lemma 3.4. ([Y]) Suppose that $X \in M_{n}(\mathbb{F})$ and $Y \in M_{s}(\mathbb{F}), 1 \leq s \leq n$ satisfy 
(a) $X+Y \oplus 0_{n-s} \in P_{n}(\mathbb{F})$;

(b) $X+\left(I_{s}+Y\right) \oplus 0_{n-s} \in P_{n}(\mathbb{F})$.

Then there are $U \in P_{s}(\mathbb{F})$ and $V \in P_{n-s}(\mathbb{F})$ such that $X=(Y+U) \oplus V$.

Lemma 3.5. Suppose that $\phi \in \Phi_{n, m}(\mathbb{F}), 1 \leq s \leq n-1$ and $r$ is a nonnegative integer satisfying $(a) \phi\left(E_{k k}\right)=I_{r} \oplus 0$ for all $k \in\{1, \ldots, n\}$, and $(b) \phi(A \oplus 0)=$ ( $\operatorname{Tr} A) I_{r} \oplus 0$ for all $A \in M_{s}(\mathbb{F})$, where $\operatorname{Tr} A$ denotes the trace of $A$. Then

$$
\phi(Z \oplus 0)=(\operatorname{Tr} Z) I_{r} \oplus 0 \text { for all } Z \in M_{s+1}(\mathbb{F}) .
$$

Proof. The proof is divided into the following four steps.

Step 1. $\phi(A \oplus \mu \oplus 0)=(\operatorname{Tr} A+\mu) I_{r} \oplus 0$ for all $A \in M_{s}(\mathbb{F}), \mu \in \mathbb{F}^{*}$.

Fix any $E \in M_{s}(\mathbb{F})$. Note that

$$
\mu^{-1}(E \oplus \mu \oplus 0)+\mu^{-1} E \oplus 0
$$

and

$$
\mu^{-1}(E \oplus \mu \oplus 0)+\left(\mu^{-1} E+1 \oplus 0\right) \oplus 0
$$

are both $n \times n$ idempotent matrices. We have by $(b)$ and $\phi \in \Phi_{n, m}(\mathbb{F})$ that

$$
\mu^{-1} \phi(E \oplus \mu \oplus 0)+\mu^{-1}(\operatorname{Tr} E) I_{r} \oplus 0 \in P_{m}(\mathbb{F})
$$

and

$$
\mu^{-1} \phi(E \oplus \mu \oplus 0)+\left(I_{r}+\mu^{-1}(\operatorname{Tr} E) I_{r}\right) \oplus 0 \in P_{m}(\mathbb{F}) .
$$

Applying Lemma 3.4 to $X=\mu^{-1} \phi(E \oplus \mu \oplus 0)$ and $Y=\mu^{-1}(\operatorname{Tr} E) I_{r}$, we see that there are $U(E, \mu) \in P_{r}(\mathbb{F})$ and $V(E, \mu) \in P_{n-r}(\mathbb{F})$ such that

$$
\phi(E \oplus \mu \oplus 0)=\left(\mu U(E, \mu)+(\operatorname{Tr} E) I_{r}\right) \oplus \mu V(E, \mu)
$$

for all $E \in M_{s}(\mathbb{F})$ and $\mu \in \mathbb{F}^{*}$.

We claim that $U(E, \mu)=I_{r}$ and $V(E, \mu)=0$ for all $E \in M_{s}(\mathbb{F})$ and $\mu \in \mathbb{F}^{*}$.

In fact, (a) tells us $U(0, \mu)=I_{r}$ and $V(0, \mu)=0$ for all $\mu \in \mathbb{F}^{*}$. Namely, the claim holds for all $E \in \Delta_{s, 0, \mu}$ and $\mu \in \mathbb{F}^{*}$. We assume that the claim is true for all $\mu \in \mathbb{F}^{*}$ and $E \in \Delta_{s, k-1, \mu}$ where $1 \leq k \leq 2 n^{2}$. Fix any $\mu \in \mathbb{F}^{*}$ and $A \in \Delta_{s, k, \mu}$. Then there are $\lambda \in F \backslash\left\{0, \mu^{-1}\right\}$ and $B \in \Delta_{s, k-1, \mu}$ such that $\lambda A+B \in P_{s}(\mathbb{F})$. Hence one 
has $\lambda(A \oplus \mu \oplus 0)+B \oplus \lambda \mu \oplus 0 \in P_{n}(\mathbb{F})$. This, together with (3.22) and the induction principle, yields that

$$
\left(\lambda \mu U(A, \mu)+\lambda(\operatorname{Tr} A) I_{r}+(\operatorname{Tr} B) I_{r}+\lambda \mu I_{r}\right) \oplus \lambda \mu V(A, \mu) \in P_{m}(\mathbb{F}) .
$$

As $\lambda \in \mathbb{F} \backslash\left\{0, \mu^{-1}\right\}$ and $\lambda \operatorname{Tr} A+\operatorname{Tr} B \in\{0,1\}$, we get $U(A, \mu)=I_{r}$ and $V(A, \mu)=0$. Now we can complete the proof of Step 1 by Lemma 2.1 and the induction principle.

$$
\text { Step 2. }\left\{\begin{array}{l}
\phi\left(\left[\begin{array}{cc}
A & \alpha \\
0 & \mu
\end{array}\right] \oplus 0\right)=(\operatorname{Tr} A+\mu) I_{r} \oplus 0 \\
\phi\left(\left[\begin{array}{cc}
A & 0 \\
\alpha^{T} & \mu
\end{array}\right] \oplus 0\right)=(\operatorname{Tr} A+\mu) I_{r} \oplus 0
\end{array} \quad \text { for all } A \in M_{s}(\mathbb{F}), \alpha \in\right.
$$

$\mathbb{F}^{s} \backslash\{0\}$ and $\mu \in \mathbb{F}$.

We only prove the first one, and the proof of the second is similar.

When $s=1$ and $\mu=0$, we know by (a) and Lemma 3.2 that

$$
\phi\left((A+1) E_{11}+E_{33}\right)=(A+1) \phi\left(E_{11}\right)+\phi\left(E_{33}\right)=A I_{r} \oplus 0 .
$$

Because $\left[\begin{array}{cc}A & \alpha \\ 0 & 0\end{array}\right] \oplus 0+\left[\begin{array}{cc}A+1 & 0 \\ 0 & 0\end{array}\right] \oplus 0$ and $\left[\begin{array}{cc}A & \alpha \\ 0 & 0\end{array}\right] \oplus 0+\left[\begin{array}{cc}A+1 & 0 \\ 0 & 0\end{array}\right] \oplus 1 \oplus 0$ are both $n \times n$ idempotent matrices, one can obtain by $(a),(3.23)$ and $\phi \in \Phi_{n, m}(\mathbb{F})$ that

$$
\phi\left(\left[\begin{array}{cc}
A & \alpha \\
0 & 0
\end{array}\right] \oplus 0\right)+(A+1) I_{r} \oplus 0 \in P_{m}(\mathbb{F})
$$

and

$$
\phi\left(\left[\begin{array}{cc}
A & \alpha \\
0 & 0
\end{array}\right] \oplus 0\right)+A I_{r} \oplus 0 \in P_{m}(\mathbb{F}) .
$$

This, together with Lemma 3.4, tells us that there are $U_{1} \in P_{r}(\mathbb{F})$ and $V_{1} \in P_{m-r}(\mathbb{F})$ such that

$$
\phi\left(\left[\begin{array}{cc}
A & \alpha \\
0 & 0
\end{array}\right] \oplus 0\right)=\left(U_{1}+A I_{r}\right) \oplus V_{1}
$$

Take $\lambda \neq 0,1$. Note that $\lambda\left[\begin{array}{cc}A & \alpha \\ 0 & 0\end{array}\right] \oplus 0+\left[\begin{array}{cc}\lambda A & 0 \\ 0 & 1\end{array}\right] \oplus 0 \in P_{n}(\mathbb{F})$. We have by (3.24), Step 1 and $\phi \in \Phi_{n, m}(\mathbb{F})$ that $\left(\lambda U_{1}+I_{r}\right) \oplus \lambda V_{1} \in P_{m}(\mathbb{F})$. Thus, one has $U_{1}=0$ and $V_{1}=0$, proving Step 2 in this case. 
When $s=1$ and $\mu \neq 0$, we know by Lemma 3.2 and (a) that

$$
\phi\left(\mu^{-1} A E_{11}+E_{33}\right)=\left(\mu^{-1} A\right) \phi\left(E_{11}\right)+\phi\left(E_{33}\right)=\left(\mu^{-1} A+1\right) I_{r} \oplus 0 .
$$

Since

$$
\mu^{-1}\left[\begin{array}{cc}
A & \alpha \\
0 & \mu
\end{array}\right] \oplus 0+\left[\begin{array}{cc}
\mu^{-1} A & 0 \\
0 & 0
\end{array}\right] \oplus 0
$$

and

$$
\mu^{-1}\left[\begin{array}{cc}
A & \alpha \\
0 & \mu
\end{array}\right] \oplus 0+\left[\begin{array}{cc}
\mu^{-1} A & 0 \\
0 & 0
\end{array}\right] \oplus 1 \oplus 0
$$

are both $n \times n$ idempotent matrices, we can get by $(a),(3.25)$ and $\phi \in \Phi_{n, m}(\mathbb{F})$ that

$$
\mu^{-1} \phi\left(\left[\begin{array}{cc}
A & \alpha \\
0 & \mu
\end{array}\right] \oplus 0\right)+\mu^{-1} A I_{r} \oplus 0 \in P_{m}(\mathbb{F})
$$

and

$$
\mu^{-1} \phi\left(\left[\begin{array}{cc}
A & \alpha \\
0 & \mu
\end{array}\right] \oplus 0\right)+\left(\mu^{-1} A+1\right) I_{r} \oplus 0 \in P_{m}(\mathbb{F})
$$

Using Lemma 3.4, we see that there are $U_{2} \in P_{r}(\mathbb{F})$ and $V_{2} \in P_{m-r}(\mathbb{F})$ such that

$$
\phi\left(\left[\begin{array}{cc}
A & \alpha \\
0 & \mu
\end{array}\right] \oplus 0\right)=\left(\mu U_{2}+A I_{r}\right) \oplus \mu V_{2} .
$$

Take $\lambda \neq 0, \mu^{-1}$. Since $\lambda\left[\begin{array}{cc}A & \alpha \\ 0 & \mu\end{array}\right] \oplus 0+\left[\begin{array}{cc}\lambda A & 0 \\ 0 & \lambda \mu+1\end{array}\right] \oplus 0 \in P_{n}(\mathbb{F})$, we have by (3.26), Step 1 and $\phi \in \Phi_{n, m}(\mathbb{F})$ that $\left(\lambda \mu U_{2}+(\lambda \mu+1) I_{r}\right) \oplus \lambda \mu V_{2} \in P_{m}(\mathbb{F})$. Thus, one has $U_{2}=I_{r}$ and $V_{2}=0$, proving Step 2 in the case $s=1$.

When $s \geq 2$, it follows from $\alpha \neq 0$ that there is an invertible matrix $Q_{\alpha} \in M_{s}(\mathbb{F})$ satisfying $\alpha=Q_{\alpha} e_{1}$. Since

$$
\left[\begin{array}{cc}
A & \alpha \\
0 & \mu
\end{array}\right] \oplus 0+\left[\begin{array}{cc}
A+Q_{\alpha}(1 \oplus 0) Q_{\alpha}^{-1} & 0 \\
0 & \mu
\end{array}\right] \oplus 0
$$

and

$$
\left[\begin{array}{cc}
A & \alpha \\
0 & \mu
\end{array}\right] \oplus 0+\left[\begin{array}{cc}
A+Q_{\alpha}(1 \oplus 1 \oplus 0) Q_{\alpha}^{-1} & 0 \\
0 & \mu
\end{array}\right] \oplus 0
$$


are both $n \times n$ idempotent matrices, one can obtain by Step 1 and $\phi \in \Phi_{n, m}(\mathbb{F})$ that

$$
\phi\left(\left[\begin{array}{cc}
A & \alpha \\
0 & \mu
\end{array}\right] \oplus 0\right)+(\operatorname{Tr} A+\mu+1) I_{r} \oplus 0 \in P_{m}(\mathbb{F})
$$

and

$$
\phi\left(\left[\begin{array}{cc}
A & \alpha \\
0 & \mu
\end{array}\right] \oplus 0\right)+(\operatorname{Tr} A+\mu) I_{r} \oplus 0 \in P_{m}(\mathbb{F})
$$

Due to Lemma 3.4, there are $U_{3} \in P_{r}(\mathbb{F})$ and $V_{3} \in P_{m-r}(\mathbb{F})$ such that

$$
\phi\left(\left[\begin{array}{cc}
A & \alpha \\
0 & \mu
\end{array}\right] \oplus 0\right)=\left(U_{3}+(\operatorname{Tr} A+\mu) I_{r}\right) \oplus V_{3}
$$

Take $\lambda \neq 0,1$. As $\lambda\left[\begin{array}{cc}A & \alpha \\ 0 & \mu\end{array}\right] \oplus 0+\left[\begin{array}{cc}\lambda A & 0 \\ 0 & \lambda \mu+1\end{array}\right] \oplus 0 \in P_{n}(\mathbb{F})$, we have by (3.27), Step 1 and $\phi \in \Phi_{n, m}(\mathbb{F})$ that $\left(\lambda U_{3}+I_{r}\right) \oplus \lambda V_{3} \in P_{m}(\mathbb{F})$. Further, we have $U_{3}=0$ and $V_{3}=0$. The proof of Step 2 is completed.

Step 3. $\phi\left(\left[\begin{array}{cc}A & \alpha \\ \beta^{T} & 0\end{array}\right] \oplus 0\right)=(\operatorname{Tr} A) I_{r} \oplus 0$ for all $A \in M_{s}(\mathbb{F}), \alpha, \beta \in \mathbb{F}^{s} \backslash\{0\}$.

If we prove that for any $A \in M_{s}(\mathbb{F}), \alpha \in \mathbb{F}^{s} \backslash\{0\}$, there are $U_{4} \in P_{r}(\mathbb{F})$ and $V_{4} \in P_{m-r}(\mathbb{F})$ such that

$$
\phi\left(\left[\begin{array}{cc}
A & \alpha \\
\beta^{T} & 0
\end{array}\right] \oplus 0\right)=\left(U_{4}+(\operatorname{Tr} A) I_{r}\right) \oplus V_{4}
$$

and then we take $\lambda \neq 0,1$, by $\lambda\left[\begin{array}{cc}A & \alpha \\ \beta^{T} & 0\end{array}\right] \oplus 0+\left[\begin{array}{cc}\lambda A & \lambda \alpha \\ 0 & 1\end{array}\right] \oplus 0 \in P_{n}(\mathbb{F})$, we can use $\phi \in \Phi_{n, m}(\mathbb{F})$ with Step 2 and $(3.28)$ to get $U_{4}=0$ and $V_{4}=0$, proving Step 3 .

To prove (3.28), we first consider the case $s=1$.

Since $\left[\begin{array}{cc}A & \alpha \\ \beta^{T} & 0\end{array}\right] \oplus 0+\left[\begin{array}{cc}A+1 & \alpha \\ 0 & 0\end{array}\right] \oplus 0$ and $\left[\begin{array}{cc}A & \alpha \\ \beta^{T} & 0\end{array}\right] \oplus 0+\left[\begin{array}{cc}A+1 & \alpha \\ 0 & 0\end{array}\right] \oplus 1 \oplus 0$ are $n \times n$ idempotent matrices, we can use Lemma 3.2, Step 2, Lemma 3.4 and $\phi \in \Phi_{n, m}(\mathbb{F})$ to get $(3.28)$.

Consider the case $s \geq 2$. It follows from $\beta \neq 0$ that there is an invertible matrix $Q_{\beta} \in M_{s}(\mathbb{F})$ satisfying $\beta=Q_{\beta} e_{1}$. Since

$$
\left[\begin{array}{cc}
A & \alpha \\
\beta^{T} & 0
\end{array}\right] \oplus 0+\left[\begin{array}{cc}
A+\left(Q_{\beta}(1 \oplus 0) Q_{\beta}^{-1}\right)^{T} & \alpha \\
0 & 0
\end{array}\right] \oplus 0
$$


and

$$
\left[\begin{array}{cc}
A & \alpha \\
\beta^{T} & 0
\end{array}\right] \oplus 0+\left[\begin{array}{cc}
A+\left(Q_{\beta}(1 \oplus 1 \oplus 0) Q_{\beta}^{-1}\right)^{T} & \alpha \\
0 & 0
\end{array}\right] \oplus 0
$$

are both $n \times n$ idempotent matrices, we see by Step 2, $\phi \in \Phi_{n, m}(\mathbb{F})$ and Lemma 3.4 that (3.28) holds.

Step 4. $\phi\left(\left[\begin{array}{cc}A & \alpha \\ \beta^{T} & \mu\end{array}\right] \oplus 0\right)=(\operatorname{Tr} A+\mu) I_{r} \oplus 0$ for all $A \in M_{s}(\mathbb{F}), \alpha, \beta \in \mathbb{F}^{s} \backslash\{0\}$ and $\mu \in \mathbb{F}^{*}$.

Note that

$$
\mu^{-1}\left[\begin{array}{cc}
A & \alpha \\
\beta^{T} & \mu
\end{array}\right] \oplus 0+\mu^{-1}\left[\begin{array}{cc}
A & \alpha \\
\beta^{T} & 0
\end{array}\right] \oplus 0 \in P_{n}(\mathbb{F})
$$

and

$$
\mu^{-1}\left[\begin{array}{cc}
A & \alpha \\
\beta^{T} & \mu
\end{array}\right] \oplus 0+\mu^{-1}\left[\begin{array}{cc}
A+\mu \oplus 0 & \alpha \\
\beta^{T} & 0
\end{array}\right] \oplus 0 \in P_{n}(\mathbb{F})
$$

This, together with $\phi \in \Phi_{n, m}(\mathbb{F})$ and Step 3, gives that

$$
\mu^{-1} \phi\left(\left[\begin{array}{cc}
A & \alpha \\
\beta^{T} & \mu
\end{array}\right] \oplus 0\right)+\left(\mu^{-1} \operatorname{Tr} A\right) I_{r} \oplus 0 \in P_{m}(\mathbb{F})
$$

and

$$
\mu^{-1} \phi\left(\left[\begin{array}{cc}
A & \alpha \\
\beta^{T} & \mu
\end{array}\right] \oplus 0\right)+\left(\mu^{-1} \operatorname{Tr} A+1\right) I_{r} \oplus 0 \in P_{m}(\mathbb{F}) .
$$

Applying Lemma 3.4 to $X=\mu^{-1} \phi\left(\left[\begin{array}{cc}A & \alpha \\ \beta^{T} & \mu\end{array}\right] \oplus 0\right)$ and $Y=\left(\mu^{-1} \operatorname{Tr} A\right) I_{r}$, we see that there are $U_{5} \in P_{r}(\mathbb{F})$ and $V_{5} \in P_{m-r}(\mathbb{F})$ such that

$$
\phi\left(\left[\begin{array}{cc}
A & \alpha \\
\beta^{T} & \mu
\end{array}\right] \oplus 0\right)=\left(\mu U_{5}+(\operatorname{Tr} A) I_{r}\right) \oplus \mu V_{5} .
$$

Take $\lambda \neq 0, \mu^{-1}$. Note that $\lambda\left[\begin{array}{cc}A & \alpha \\ \beta^{T} & \mu\end{array}\right] \oplus 0+\left[\begin{array}{cc}\lambda A & \lambda \alpha \\ 0 & \lambda \mu+1\end{array}\right] \oplus 0 \in P_{n}(\mathbb{F})$. We have by (3.29), Step 2 and $\phi \in \Phi_{n, m}(\mathbb{F})$ that $\left(\lambda \mu U_{5}+(\lambda \mu+1) I_{r}\right) \oplus \lambda \mu V_{5} \in P_{m}(\mathbb{F})$. Thus, we get $U_{5}=I_{r}$ and $V_{5}=0$. The proof of Lemma 3.5 is completed. $\square$

Lemma 3.6. Suppose that $\phi \in \Phi_{n, m}(\mathbb{F})$. Define a map $\psi$ from $M_{n}(\mathbb{F})$ to $M_{m}(\mathbb{F})$ by $\psi(A)=\phi(A)+(\operatorname{Tr} A) I_{m}$ for all $A \in M_{n}(\mathbb{F})$. Then $\psi \in \Phi_{n, m}(\mathbb{F})$. 
Proof. If $A+\lambda B \in P_{n}(\mathbb{F})$, where $A, B \in M_{n}(\mathbb{F})$ and $\lambda \in \mathbb{F}$, then one has $\phi(A)+\lambda \phi(B) \in P_{m}(\mathbb{F})$ and $\operatorname{Tr} A+\lambda \operatorname{Tr} B=\operatorname{Tr}(A+\lambda B) \in\{0,1\}$. We deduce $\phi(A)+\lambda \phi(B)+(\operatorname{Tr} A+\lambda \operatorname{Tr} B) I_{m} \in P_{m}(\mathbb{F})$. This implies that $\psi(A)+\lambda \psi(B) \in$ $P_{m}(\mathbb{F})$.

4. The main result and remark. Our main result is the following.

THEOREM 4.1. Suppose $\mathbb{F} \neq \mathbb{F}_{2}$ is any field of characteristic $2, n$ and $m$ are integers with $n \geq m$ and $n \geq 3$. Then $\phi \in \Phi_{n, m}(\mathbb{F})$ if and only if there is an invertible matrix $T \in M_{m}(\mathbb{F})$ such that one of the following cases holds.

(a) $m=n$ and $\phi(A)=T A T^{-1}$ for all $A \in M_{n}(\mathbb{F})$;

(b) $m=n$ and $\phi(A)=T A^{T} T^{-1}$ for all $A \in M_{n}(\mathbb{F})$;

(c) $m=n$ and $\phi(A)=T A T^{-1}+(\operatorname{Tr} A) I_{m}$ for all $A \in M_{n}(\mathbb{F})$;

(d) $m=n$ and $\phi(A)=T A^{T} T^{-1}+(\operatorname{Tr} A) I_{m}$ for all $A \in M_{n}(\mathbb{F})$;

(e) $\phi(A)=T\left((\operatorname{Tr} A) I_{r} \oplus 0_{m-r}\right) T^{-1}$ for all $A \in M_{n}(\mathbb{F})$, where $r \in\{0,1, \ldots, m\}$ is an integer.

Proof. The proof of the "if" part is obvious. Now we prove the "only if" part.

By Lemma 3.3, we know that there exists an invertible matrix $T \in M_{m}(\mathbb{F})$ such that $\phi$ satisfies one of the condition in Lemma 3.3. If $\phi$ satisfies the Condition $(a)$ of Lemma 3.3, then [7] tells us that $\phi$ is of the form $(a)$ or $(b)$. Similarly, if $\phi$ satisfies the condition $(c)$ of Lemma 3.3, then we see by the induction principle and Lemma 3.5 that $\phi$ is of the form $(e)$.

Now we assume that $\phi$ satisfies the condition $(b)$ of Lemma 3.3. Define a map $\psi$ from $M_{n}(\mathbb{F})$ to $M_{m}(\mathbb{F})$ is given by $\psi(A)=\phi(A)+(\operatorname{Tr} A) I_{m}$ for all $A \in M_{n}(\mathbb{F})$. Then we have from Lemma 3.6 that $\psi \in \Phi_{n, m}(\mathbb{F})$. But it is not difficult to check that $\psi$ satisfies the condition $(a)$ of Lemma 3.3. So $\psi$ is of the form $(a)$ or $(b)$. This implies that $\phi$ has the forms $(c)$ or $(d)$.

REMARK 4.2. We give an example for which $n=2$ and $\phi \in \Phi_{n, m}(\mathbb{F})$. Let $\phi$ be a map from $M_{2}(\mathbb{F})$ to itself given by

$$
\phi(A)=(\operatorname{Tr} A) E_{11}+f(A) E_{12} \text { for all } A \in M_{2}(\mathbb{F}),
$$

where $f$ is a map from $M_{2}(\mathbb{F})$ to $\mathbb{F}$ satisfying

$$
f\left(\left[\begin{array}{ll}
a & b \\
c & d
\end{array}\right]\right)=\left\{\begin{array}{cl}
b, & \text { if } c=0 \\
\frac{b^{2}}{c}, & \text { if } c \neq 0 .
\end{array}\right.
$$

Then it is easy to see that $\phi \in \Phi_{2,2}(\mathbb{F})$, but $\phi$ is not linear. In fact, we see by Theorem 4.1 that $\phi$ is linear if $n \geq 3$. This shows that the same problem in the case of $n=2$ 
is complicated.

Acknowledgment. The authors would like to thank the referee for invaluable comments and suggestions on an earlier version of the paper.

\section{REFERENCES}

[1] G.H. Chan, M.H. Lim, Linear preservers on powers of matrices, Linear Algebra Appl. 162-164 (1992) 615-626.

[2] G. Dolinar, Maps on matrix algebras preserving idempotents, Linear Algebra Appl. 371 (2003) 287-300.

[3] C.K. Li, S. Pierce, Linear preserver problems, Amer. Math. Monthly 108 (2001) 591-605.

[4] S.W. Liu, Linear maps preserving idempotence on matrix modules over principal ideal domains, Linear Algebra Appl. 258 (1997), 219-231.

[5] P. Šemrl, Hua's fundamental theorems of the geometry of matrices and related results, Linear Algebra Appl. 361 (2003) 161-179.

[6] X.M. Tang, J.L. Xu, C.G. Cao, A note on idempotence-preserving maps, Linear and Multilinear Algebra 56 (2008) $399-414$.

[7] X.M. Tang, J.L. Xu, C.G. Cao, Idempotence-preserving maps on matrix spaces over field of characteristic 2, J. Natural science of Heilongjiang University, 26(2) (2009) 141-149.

[8] X. Zhang, Idempotence-preserving maps without the linearity and surjectivity assumptions, Linear Algebra Appl. 387 (2004) 167-182. 